\title{
Recovery of Cancer Patients Who Underwent Ayurvedic Palliative Treatment: Developing a New Perspective for Cancer Care
}

\author{
Sudha Karayil', Namboothiri $\mathrm{CNT}^{2}$, Parameswaran MP' and Haridas $\mathrm{M}^{3 *}$ \\ 'Integrated Rural Technology Centre, Palakkad, India \\ ${ }^{2}$ Akhilasakthy Medical Institute and Health Centre, Kottayam, India \\ ${ }^{3}$ Inter University Centre for Bioscience, Kannur University, Thalassery, India
}

\section{Abstract}

This report is the result of palliative treatment reviews of cancer patients who were, at first, treated as per the protocols of modern medicine. As all the options of modern medicine were exhausted and the patients were not shown disease-free, they were sent home for palliative care. Some of such patients were treated by a qualified physician of modern medicine using Ayurvedic medicaments generally prescribed for various symptoms of diseases, and to improve metabolic state. It is not Ayurvedic Prakriti (genetic variation) based treatment protocols. Total recovery from cancer (of various aetiologies) has been observed in the presented cases by diagnostic methods and the expressions of healthy living of the ex-patients. This report suggests a better palliative care of cancer patients and/or hypothesis for critical evaluation of Ayurvedic practices for evidences/proofs for cancer in terms of contemporary science. As well, it is suggested to assess the advantage of Ayurvedic medicines-based cancer treatment concurrent with modern medicine.

Keywords: Cancer; Ayurvedic palliative treatment; Ayurvedic medicines

\section{Introduction}

The present study is based on hypothesis that Ayurveda as a system of medical science, viewing the diseases as deviations from the expression of totality of metabolism, including the environment, and treatment as putting the system back to the normality by applying medicaments and redefining the environment. Cancer is viewed as systemic disease and various types of cancers are recognized and treatments prescribed in Ancient Ayurvedic texts [1-3]. However, the approach of Ayurveda is as a system of personalized medicine and certain types of cancers could be approached with the highlight of personalization or genomics [4]. And, Ayurveda has its own methods of practicing personalized medicine, based on its theories of systems and diseases and cancer is no exception to this practice [5-8]. Though no evidence has been generated by contemporary scientific methodologies to support Ayurvedic method as a treatment strategy for cancer, several Ayurvedic practitioners have placed on record that they had been successful in treating cancers. It offers a treatment regime as palliative mode for terminal patients, giving them, some hope to live further when all the modern medical treatments are exhausted. At this juncture there is no legal or ethical issue according

Citation: Karayil S, Namboothiri CNT, Parameswaran MP, Haridas M. Recovery of Cancer Patients Who Underwent Ayurvedic Palliative Treatment: Developing a New Perspective for Cancer Care. Ann Clin Case Stud. 2020; 2(1): 1021.

\section{Copyright: @ 2020 Sudha Karayil}

Publisher Name: Medtext Publications LLC

Manuscript compiled: Apr $15^{\text {th }}, 2020$

${ }^{*}$ Corresponding author: Haridas M, Inter University Centre for Bioscience, Kannur University, Thalassery Campus, Palayad-670661, Thalassery, India, E-mail: mharidasmm@gmail.com to contemporary scientific epistemology and understanding involved in adopting Ayurvedic palliative treatment.

The present study involved the treating of cancer patients who had been discharged from cancer hospitals of modern medicine after exhausting all the contemporary possibilities of treatments. Such patients were left to palliative care, waiting for their last moments. Some of those patients were treated by a qualified physician from the stream of modern medicine using Ayurvedic medicaments, generally prescribed for various symptoms of diseases; but not based on Ayurvedic treatment protocols. The changes in certain, available patients could be reviewed, since they showed improvements in the quality of their lives. The study is presented below.

\section{Materials and Method of Palliative Cancer} Care

\section{Hypothesis}

It is natural for a cancer patient to seek a different treatment regime when sent home from hospital after treatment programmes of modern medicine. The main aim of the patients/relatives would be to alleviate the side effects of modern medical treatment. Patients prefer to try the Ayurvedic system to treat side effects where Ayurveda is a medical system of practice, and considered a holistic system, eliciting little side effects. This preference owes more to faith than evidence. However, Ayurveda has no definite protocol for addressing diverse demands of cancer patients sent home from hospitals after treatment. The fundamental, personalised approach of Ayurvedic system has a complex scenario [1]. And, the Ayurvedic physicians are not specifically trained to treat such patients. This has hampered the development of an Ayurvedic treatment protocol for cancer patients. In the search for treatment protocols and physicians addressing the palliative care of cancer patients, the second author of this article (CNT) got teamed up with the other authors to look critically at the results of Ayurvedic palliative care of cancer patients. CNT, a surgeon 
of the modern medical stream, has a record of treating such cancer patients for thirty plus years. He follows the protocol developed/ advised to perform universally, by two great Ayurvedic physicians of yester years, famous for their clinical skills*. The protocol with check points devised by them does not adhere to the Ayurvedic system of personalised medicine. They devised a protocol with Ayurvedic medicines to apply on all palliative-care-cancer-patients without considering the prakriti of the patients $[1,2]$. This system made possible for CNT to practice and review the results now. The present analysis of clinical records of Ayurvedic palliative care of cancer patients would help develop a structured study for the benefit of posterity.

\section{Data}

The present analysis is limited to the data pertaining to the palliative-cared cancer patients of the "co-author's patient-treatment history records.

\section{Limitations of data}

This is not a conducted study. It is only the analysis of improvements observed in Ayurvedic-palliative-cared cancer patients. It is to be stated here by observing many partially successful cases, that there might have been many more successful cases of palliative Ayurvedictreatments. The missing data could be due to non-reported death of patient, ignorance of patients (and relatives of patients), or any other reason by which patients didn't turn up for continuing treatment. The Ayurvedic-palliative-care treatment protocol and medicines are provided below.

The approach was to provide drugs based on Ayurvedic theory, by combining herbs and other time-tested suitable materials to achieve the following:

1. Regain healthy metabolism as understood by indicators of good health, such as appetite for food, normal defecation and other excretory processes, and proper sleep

2. Arrest the growth of disease expressed

3. Address elimination of the possible causes of the disease

Drugs were formulated based on Ayurvedic theory of balancing tridosha, to achieve all the three aims given above simultaneously. Drugs were formulated by addition, deletion or as prescribed in ancient formulae for balancing tridosha or utilizing the herbs independently studied by contemporary scientific methods providing evidence [9-16]. They are as follows:

\section{Thathwamacy}

\section{Containing:}

Glycyrrhiza uralenis [17]

Aerva sanguimolenta [18],

Nalpamaraadi [10]

Ficus benghalensis

Ficus religiosa

Ficus lacor

Ficus glomerata

Terminalia chebula,

Terminalia bellirica,
Emblica officinalis

Santalum album

Vetiveria zizanioides

Saussurea lappa

Rubia cordifolia

Angelica glauca

Curcuma longa

Fumaria indica

Varanadi [9]

Crataeva religiosa

Strobilanthes ciliates

Asparagus racemosus

Plumbago zeylanica

Chenomorpha fragrans

Aegle marmelos

Aristolochia bracteplate

Solanum indicum

Aerua lanata

Pongamia glabra

Holoptelia integrifolia

Premna corymbosa

Terminalia chebula

Moringa olifera

Desmostachya bipinnata

Semicarpus anacardium

\section{NLP powder}

Made of barks of the following trees:

Ficus benghalensis [19]

Ficus religiosa [20]

Ficus lacor [21]

Ficus glomerata [22]

\section{Chest extract}

Containing the exact of

Aerva sanguimolenta [18]

\section{Wilwax extract}

Extract of Aegle marmelos [23]

\section{Elenji extracts}

Mimusops elenji [24]

\section{DPL syrup or capsule}

Contains the extracts of Wrightia tinctoria [25] 


\section{Salpenia}

Seeds of Caesalpinia bonducella

Asoka gritham [27]

Gandha thailam [9]

Ashtangahrudayam Uttarasthana 27/36-41

Rasnadi Kashayam [10] (Sahasrayogam, Churnaprakaranam)

Routine chemico-pathological tests, diagnostic imaging and clinical assessments were performed at the start of the treatment to assess the condition of the patients. Serum amylase was considered as the single most important parameter for assessing the condition of the patients for evidence. Drug dosages were continued in Ayurvedic format for sixty days [28]. The assessment of treatment was done in phases of sixty days for considering drug dosage changes. If no improvement was evidenced, the first phase was continued for another sixty days. This assessment was continued till the serum amylase value got lowered below 100 by the treatment. If it is below 100 , then the specific drug, Shilajit would be started along with the other drugs 1 to 8 given above. The dosages of the 1 to 8 drugs may be regulated as per the clinical wisdom of the physician in the next phase of treatment with Shilajit [29-32].

Processed Shilajit, prepared by the Ayurvedic method, namely Navakam is the drug of choice to ameliorate cancer by the ancient wisdom. Many fold purification of Shilajit is carried out [31,32]. The dose is calculated according to the stage/severity of the disease. The duration of this treatment would depend upon the severity of the disease. It may have to be continued for a few years if necessary.

If the patient-body accepts the medicines as evidenced by continued improvement of patient's condition, both physical and mental status shown by laboratory and clinical examinations, a total rolling back to normalcy may be expected.

Throughout the course the patients are restrained from smoking, alcohol, difficult-to-digest food, etc. Maintenance of the general health, by symptomatic supportive physiotherapy treatments if necessary should be done throughout the course of the treatment.

The following medicines are used according to the severity of disease. The treatment is conceived in phases, as follows:

$I^{\text {st }}$ Phase: Duration 2-4 months (60 to 120 days): Drugs 1-11

II ${ }^{\text {nd }}$ Phase: Duration 6 months: ${ }^{\text {st }}$ Phase Drugs, with the addition of Navakam $(32,33)$

III ${ }^{\text {rd }}$ Phase: Duration 6-12 months

IV ${ }^{\text {th }}$ Phase: 16 months of preventive and Rejuvenation time

$\mathbf{I}^{\text {st }}$ Phase Treatment: All the eleven medicines described above are prescribed to any patient coming forward for Ayurvedic palliative treatment. Dosage would be made considering age, sex, serum amylase level and other clinical features of the patients. In addition to the eleven medicines listed above other Ayurvedic medicines may also be prescribed to put the metabolic state of the patient back to normal. There is no single clinical test to establish that the metabolism is in order. It is a primary concept in Ayurveda that the orderliness of digestive system is an indicator of good health. Pancreatitis of various etiologies, tumors, gall bladder infection, kidney failure, etc. may result in hyperamylasemia [1]. Since hyperamylasemia being clinically very important, the patients undergoing Ayurvedic palliative care are tested for serum amylase, a bench mark of health status, conforming to the Ayurvedic concepts also. The status of serum amylase would serve as a good clinical record of monitoring the improvement brought by medication.

II $^{\text {nd }}$ Phase Treatment: After 60/120 days of treatment, Serum Amylase is to be assessed. If it is below 100, Navakam is added with the drugs of $I^{\text {st }}$ phase for the treatment $[31,32]$. This preparation, purified shilajit is considered to be anti-cancer as per Ayurvedic texts and has been proved to be correct. Shilajit or mumijo is claimed to have anti cancer properties together with a whole lot of claims of other cures, including phthisis [2-5]. It contains hundreds of natural compounds, both biological and mineralogical. Evidences to the anticancer property of shilajit have been already established [32]. At the 5th month of Navakam treatment, Liver function and general conditions have to be assessed by laboratory methods. At 6th month thyroid function tests must be done to indicate general health.

III $^{\text {rd }}$ Phase of Treatment: Tapering of the medicines would be considered at this stage. The process will be continued for 6 months. All the relevant investigations, including blood analyses, X-Rays, MRI scanning, etc. may be considered for complete withdrawal of the medicines. If everything is satisfactory then the medicines may be stopped.

IV $^{\text {th }}$ Phase of Treatment: This phase is meant to improve body and mind status back to normalcy by rejuvenating medicines, nutraceuticals and counselling.

\section{Summary of Cases}

\section{Case 1 (@Supplementary data 1.1.a-c, 1.2.a-c, 1.3)}

A five year old girl was having complaints of progressive difficulty in walking for past one month, and diagnosed to have a right lateral ventricular atypical teratoid rhabdoid tumour (WHO grade IV) and a right parieto-occipetal craniotomy and radical excision was done for it on the $14^{\text {th }}$ August-2013 at Christian Medical College, Vellore, India. Postoperatively she received craniospinal irradiation in September 2013, and subsequently received four cycles of carboplatin and etoposide chemotherapy, which she completed in March 2014. There was no symptom suggestive of raised intracranial pressure or any behavioural changes. She did not have any vision complaints. She was evaluated elsewhere and detected to have a cervical intra medullar lesion, for which she was given treatment with steroids and her condition did not improve.

Whole body PET scan done on 01 September, 2014 showed a metabolically active lesion in the cervical cord, probably tumour recurrence. There was also increased metabolic activity seen in the mid thoracic and lower thoracic cord. Repeat MRI scan was done 03 September, 2014 showed that the size of the contrast enhancing lesion remained unchanged. The CSF analysis was normal and did not reveal any infectious features. The PET scan showed increased metabolic activity at the cervical cord, corresponding to the intra medullary lesion. A repeat MRI showed mild reduction in the oedema in the cervical cord, implicated to the steroids she was receiving. But the size of the tumour remained the same. It was considered as the lesions were metastatic from its diffuse nature and inoperable. No chemotherapy or radiotherapy further was considered possible to improve the state of the child and advised for palliative treatment only. She was discharged on a tapering course of steroids, with little further treatment options. 
At this stage she was taken to CNT at the end of 2014 for further treatment. She was given the treatment at CNT's as described in the Methodology above. She was treated for one and half years to get tapered off all the medicines, as she improved. Now she leads a normal healthy life.

Case 2 (@Supplementary data 2.1, 2.2, 2.3, 2.4.a-b, 2.5, 2.6.ab, 2.7, 2.82.9, 2.10.a-d, 2.11)

In April, 2011 a twenty-year-old female reported at Al Hilal Hospital, Bahrain with a six years history of pain in both legs with severe pain on left thigh. It was diagnosed as myoma and suggested to be operated immediately. She approached Cochin Hospital, Kochi, India and underwent excision of the lesion on the $30^{\text {th }}$ April, 2011. Biopsy examination showed no malignancy and the nature of the solid mass was not confirmed. It was said to be lipoma only. After surgery the condition became better (Patient reported).

The patient started experiencing pain after three months of surgery. In September, 2011 MRI was done and the reports showed its recurrence and found it encased in sciatic nerve. The patient approached Lake Shore Hospital, Kochi, India. CT angiogram done at Lakeshore Hospital, on 01.10.2011 showed evidence of recurrent residual soft tissue in left upper thigh and the knee. They asked for a second opinion at Amrutha Hospital, Kochi, India. Later in October, 2011, the Amrutha Hospital referred the case to Tata Memorial Hospital (TMH), Mumbai, India. On 05.11.2011 she underwent multiplanar CEMRI scan at Elite Mission Hospital, Thrissur revealed both thighs including knee joint showed confirmed existence of the soft tissue lesions $(12.3 \mathrm{~cm} \times 7.6 \mathrm{~cm} \times 5 \mathrm{~cm})$ in the left adductor brevis and Magnus muscles. On 19.11.2011 histopathology reports -multiple sections examined showed features of fibromatosis at TMH. In January, 2012, chemotherapy was started at Al Hilal Hospital, Bahrain as advised by the TMH. The patient's condition worsened and she became almost immobile and could not attend school for nearly one year. The patient was brought back to Kerala.

On 16.01.2013 MRI was done which documented a large inter muscular lesion in the posterior aspect of the thigh measuring approximately $25.7 \mathrm{~cm} \times 8.3 \mathrm{~cm} \times 5.2 \mathrm{~cm}$ in size which showed no significant change in the size or morphological appearance since the previous examination dated September 2012, suggesting recurrent fibromatosis.

In April, 2014, as her condition worsened, she was taken to CNT. She was given the treatment at CNT's since then as described in the Methodology above. Within a few months of the palliative treatment at CNT the pain and side effects of chemotherapy disappeared. The palliative treatment by CNT continued for more than three years. MRI results showed a decrease in the size of mass and hardness. She was tapered off all the medicines, as she improved. Now she leads a normal healthy life.

\section{Case 3 (@Supplementary data 3.1.a-d, 3.2, 3.3.a-b)}

Eight-year-old boy complained of excessive sweating, vomiting dizziness, severe headache and blurred vision and was ultimately diagnosed as having a tumour in the cerebellar pilocytic astrocytoma brain. At the age of ten in 2008 he underwent surgery, radiation, and no chemotherapy was given. Later in 2008, recurrence of tumour was found with left arm tremor. Treatment with CNT started on advice from friends. He was given the treatment at CNT's as described in the Methodology above. Today he is free from most of the complaints. The MRI scan showed non-reactive residual lesion. His general condition is normal.

\section{Discussion}

The cases reported here showed remarkable difference before and after their Ayurvedic palliative treatment. Total recovery from cancer (of various aetiologies) has been observed in the presented cases by diagnostic methods and the expressions of healthy living of the expatients. Essentially, the present report is not based on a programmed study. One of the authors (CNT) has been treating cancer patients who had exhausted with all possible, available methods of cancer treatment and sent out from hospitals with advice to follow palliative treatments of any systems of medicine, including Ayurveda. This is to reiterate that none of these patients at any stage of their disease condition had resorted to any method of treatment other than modern medicine. It may be noted that, though hundreds of patients have been given palliative treatment as described in the methodology of treatment by CNT during nearly two decades of this century, only a few of the patients/their relatives were available and cooperative for sparing their treatment records and other information. Obviously, most of the patients at the terminal stage of their life would not have presence of mind and optimism to share data of their illness.

\section{Conclusion}

The analyses of the cases referred to by providing proof of total recovery from malignancy demonstrate that the line of palliative treatment signifies more than palliative and tends towards real treat of cancer at least in certain cases. It is to be assumed that these patients would not have had the chance of having a better and longer life after the exhaustion of protocols of modern medicine. Certainly, this analysis is not suggestive to the extent that the line of treatment given in this article as an independent treatment line, better than the existing evidence based treatment protocols. However, the treatment line described in this article is worthy of more rigorous, planned investigation since it is based on Ayurvedic treatment methodologies which produce very good results, at present at least in certain cases with no hope. The Ayurvedic model may be assumed like a play of multiple seesaws anchored at one point with natural ligands/drugs on one side and their target proteins on the other side. The player pairs who are able to down play others shall cause imbalance in their systems biology expression leading to disease. Disease amelioration would be controlling the violent players causing physiological imbalance. Treatment in Ayurvedic format would lead to normalize multi factorial imbalances including cancers. Combined application of network pharmacology (synergistically back and forth to get intelligent mixtures), systems biology and bioinformatics may provide evidence in this regard. It may help improve the present format of Ayurvedic palliative treatment and instill confidence among the cancer patients who are at the commencing palliative treatment. It may also help improve the present regime of cancer treatment, particularly in reducing the side effects of cancer treatment if applied early. As well, it is suggested to assess the advantage of Ayurvedic medicines-based cancer treatment concurrent with modern medicine.

\section{Acknowledgements}

The authors are extremely grateful to the three patients who practically recovered from cancer and side effects of cancer treatment and their relatives for kindly giving permission to publish their treatment records. This study has been funded by the Unnimmose Foundation, Thrissur, Kerala, India. Also, authors acknowledge Dr. N Devan, Asokalayam, Thiruvegappura, Kerala, India for the stimulating discussions and encouragements. 


\section{Author Contributions}

MMP: Developed the idea, procured the financial support for SK, designed the study and co-wrote the manuscript with $\mathrm{MH}$.

NCNT: Treated (palliative) the cancer patients and participated in study.

SK: Involved in developing the idea, collected presentation and wrote data and participated in study.

$\mathrm{MH}$ : Involved in designing the study and prepared the frame of the manuscript.

\#Vaidyamadhom Cheriya Narayanan Nampoothiri and Vaidyabhooshanam Raghavan Thirumulpad (online sources).

@Supplementary data 1-3, DOI: http://dx.doi.org/10.33597/2688-

\section{1-v2-id1021}

\section{References}

1. Prasad GC, Sahu M, Deshpande PJ. Concept of cancer in Ayurveda. Ancient Science of Life. 1982;1(3):172-6.

2. Singh RH. An Assessment of the Ayurvedic Concept of Cancer and a New Paradigm of Anticancer Treatment in Ayurveda. J Altern Complement Med. 2002;8(5):609-14.

3. Balachandran P, Govindarajan R. Cancer-an ayurvedic perspective. Pharmacol Res. 2005;51(1):19-30.

4. Chin L, Andersen JN, Futreal PA. Cancer genomics: from discovery science to personalized medicine. Nat Med. 2011;17(3):297-303

5. Sumantran VN, Tillu G. Ayurvedic pharmaceutics and insights on personalized medicine. India: Traditional and Folk Herbal Medicine; 2011

6. Chatterjee B, Pancholi J. Prakriti-based medicine: A step towards personalized medicine. Ayu. 2011;32(2):141-6

7. Govindaraj P, Nizamuddin S, Sharath A, Jyothi V, Rotti H, Raval R, et al. Genomewide analysis correlates Ayurveda Prakriti. Sci Rep. 2015;5:15786.

8. Richardson MA, Sanders T, Palmer JL, Greisinger A, Singletary SE. Complementary/ Alternative Medicine Use in a Comprehensive Cancer Center and the Implications for Oncology. J Clin Oncol. 2000;18(13):2505-14.

9. Srikantha Murthy KR. Vagbhata's Astanga Hrdayam. Chaughamba Orientalia. India. 2014.

10. Prabhakara Rao G. Sahasrayogam: Compendium of 1000 Ayurvedic Formulations, India: Chaughamba Orientalia; 2016

11. Dey S, Pahwa P. Prakriti and its associations with metabolism, chronic diseases, and genotypes: Possibilities of newborn screening and a lifetime of personalized prevention. J Ayurveda Integr Med. 2014;5(1):15-24

12. Sharma H, Clark C. Contemporary Ayurveda: Medicine and Research in Maharishi Ayur-Veda. Churchill Livingstone Press. London. 1998.

13. Prasher B, Gibson G, Mukerji M. Genomic insights into Ayurvedic and western approaches to personalized medicine. J Genet. 2016;95(1):209-28.

14. Hankey A. Ayurvedic physiology and etiology: Ayurvedo Amritanam. The doshas and their functioning in terms of contemporary biology and physical chemistry. J Alt Complement Med. 2001;7(5):567-74.

15. Hankey A. The scientific value of Ayurveda. J Alt Complement Med. 2005;11(2):2215

16. Hankey A. Establishing the Scientific Validity of Tridosha part 1: Doshas, Subdoshas and Dosha Prakritis. Anc Sci Life. 2010;29(3):6-18.

17. Shen Z, Qin Q, Liao XL,Yang B. Host-guest inclusion system of glycyrrhetic acid with polyamine- $\beta$-cyclodextrin: Preparation, characterization, and anticancer activity. J Mol Struct. 2017;1149:155-161.

18. Lalee A, Pal P, Bhattacharaya B, Samanta A. Evaluation of Anticancer activity of Aerva sanguinolenta (L.) (Amaranthaceae) on Ehrlich's Ascites cell induced Swiss Mice. Int J Drug Dev \& Res. 2012;4(1):203-9.

19. Patel R, Gautam P. Medicinal Potency of Ficus Bengalensis: A Review. Int J Med Chem. 2014;4(1):53-8.

20. Chandrasekar SB, Bhanumathy M, Pawar AT, Somasundaram T. Phytopharmacology of Ficus religiosa. Pharmacogn Rev. 2010;4(8):195-9.

21. Kalaskar MG, Surana SJ. Pharmacognostic and phytochemical studies on Ficus Microcarpa L. fil. Anc Sci Life. 2012;32(2):107-11.

22. Rahman NN, Khan M, Hasan R. Bioactive components from Ficus glomerata. Pure Appl Chem. 2009;66(10-11):2287-90.

23. Maity P, Hansda D, Bandyopadhyay U, Mishra DK. Biological activities of crude extracts and chemical constituents of Bael, Aegle marmelos (L.) Corr. Indian J Exp Biol. 2009;47(11):849-61.

24. Gami B, Pathak S, Parabia M. Ethnobotanical, phytochemical and pharmacological review of Mimusops elengi Linn. Asian Pac J Trop Biomed. 2012;2(9):743-8.

25. Bigoniya P, Singh CS, Shukla A. Pharmacognostical and physicochemical standardization of ethnopharmacologically important seeds of Lepidium sativum Linn. and Wrightia tinctoria R. Br. Indian J Nat Prod Resour. 2011;2(4):464-71.

26. Shukla S, Mehta A, John J, Singh S, Mehta P, Vyas SP. Antioxidant activity and tota phenolic content of ethanolic extract of Caesalpinia bonducella seeds. Food Chem Toxicol. 2009;47(8):1848-51.

27. Bhaishajya ratnavali, Prabhakara Rao G. 2014. Chaughamba Orientalia. India

28. Jha RK, Ma Q, Sha H, Palikhe M. Acute pancreatitis: a literature review. Med Sci Monit. 2009;15(7):147-56

29. Ghosal S, Chemistry of shilajit, an immunomodulatory Ayurvedic rasayan. Pure and Appl Chem. 1990;62(7):1285-88

30. Al-Himaidi AR, Umar M. Safe use of salajeet during pregnancy of female mice. J Biol Sci. 2003;3(8):681-4.

31. Mittal P, Kaushik D, Gupta V, Bansal P, Khokra S. Therapeutic Potentials of "Shilajit Rasayana”-A Review. Int J Pharm Clin Res. 2009;1(2):47-9.

32. Mirza MA, Alam MN, Faiyazuddin M, Mahmood D, Bairwa R, Mustafa G. Shilajit An Ancient Panacea. Int J Pharm Sci Rev Res. 2010;1(1):2-11. 